\title{
Turbulent cross-field transport of non-thermal electrons in coronal loops: theory and observations
}

\author{
N. H. Bian, E. P. Kontar, and A. L. MacKinnon
}

Department of Physics \& Astronomy, University of Glasgow, G12 8QQ, UK

e-mail: nicolas.bian@glasgow.ac.uk

Received 27 June 2011 / Accepted 20 August 2011

\section{ABSTRACT}

\begin{abstract}
Context. A fundamental problem in astrophysics is the interaction between magnetic turbulence and charged particles. It is now possible to use Ramaty High Energy Solar Spectroscopic Imager (RHESSI) observations of hard X-rays (HXR) emitted by electrons to identify the presence of turbulence and to estimate the magnitude of the magnetic field line diffusion coefficient at least in dense coronal flaring loops.

Aims. We discuss the various possible regimes of cross-field transport of non-thermal electrons resulting from broadband magnetic turbulence in coronal loops. The importance of the Kubo number $K$ as a governing parameter is emphasized and results applicable in both the large and small Kubo number limits are collected.

Methods. Generic models, based on concepts and insights developed in the statistical theory of transport, are applied to the coronal loops and to the interpretation of hard X-ray imaging data in solar flares. The role of trapping effects, which become important in the non-linear regime of transport, is taken into account in the interpretation of the data.

Results. For this flaring solar loop, we constrain the ranges of parallel and perpendicular correlation lengths of turbulent magnetic fields and possible Kubo numbers. We show that a substantial amount of magnetic fluctuations with energy $\sim 1 \%$ (or more) of the background field can be inferred from the measurements of the magnetic diffusion coefficient inside thick-target coronal loops.
\end{abstract}

Key words. Sun: X-rays, gamma rays

\section{Introduction}

Solar flares provide many observational challenges for crucial aspects of high-energy astrophysics, including energy release, particle acceleration and transport in magnetized plasmas. In the standard flare scenario, magnetic energy stored in the corona is released via plasma heating, bulk motions and particle acceleration. Thanks to hard X-ray (HXR) imaging spacecraft such as Yohkoh/HXT (Kosugi et al. 1991) and RHESSI (Lin et al. 2002), high-resolution spatial and spectral diagnostics of energetic particles (Shibata 1999; Aschwanden 2002; Lin et al. 2003; Brown \& Kontar 2005; Lin 2006) have proven to be vital for our understanding of the physics of the solar corona.

Turbulence, an important element of the solar flare scenario, is believed to be associated with various physical processes, from the triggering of fast magnetic reconnection to particle acceleration and transport. Many particle acceleration models rely on the presence of electromagnetic fluctuations during flares and it has been shown that stochastic acceleration can effectively energize and accelerate a large number of electrons and ions (Miller \& Ramaty 1987; Hamilton \& Petrosian 1992; Melrose 1994; Bykov \& Fleishman 2009; Petrosian \& Chen 2010; Bian et al. 2010). The precise origin of the turbulence in the acceleration region of flaring loops is still unclear but it has been suggested that it could be associated with current sheets (Chiueh \& Zweibel 1987; Somov \& Kosugi 1997; Litvinenko 2006) and/or with reconnection outflows (Larosa et al. 1994). Independently of its origin, if turbulence has a significant impact on particle acceleration, it must also be expected to manifest itself via the transport of particles, including HXR-emitting electrons. This opens the route of using HXR data to characterize turbulent processes involved in particle acceleration and transport during solar flares.

A step forward in this direction was recently taken by Kontar et al. (2011), who developed a method for determining the magnetic diffusion coefficient in flaring loops. Their approach was inspired by a study of Xu et al. (2008), which analyses the variation of the HXR source size along the guiding field of the loop as a function of energy, see also Prato et al. (2009). Specifically, Kontar et al. (2011) have shown that the size of the HXR source in the direction perpendicular to the magnetic field is also a growing function of energy. These observations strongly suggest cross-field mobility of non-thermal electrons inside flaring loops.

Owing to their high speed and small Larmor radius, the cross-field transport of energetic electrons in loops is likely to be dominated by perpendicular magnetic fluctuations and by the resulting wandering of the magnetic field lines. The perpendicular transport of magnetic field lines is usually quantified in terms of a diffusion coefficient $D_{m}$ and estimates of this diffusion coefficient were obtained in Kontar et al. (2011) through imaging observations of HXR-emitting electrons.

The magnetic diffusion coefficient $D_{m}$ depends on three quantities, which are the relative level of the turbulent magnetic fluctuations and their parallel and perpendicular correlation lengths: $B_{\perp} / B_{0}, \lambda_{z}, \lambda_{\perp}$, respectively. Because the dimension of the magnetic diffusion coefficient is a length, dimensional analysis gives that $D_{m} \approx\left(\lambda_{\perp}^{2} / \lambda_{z}\right) K^{\gamma}$. The number $K$ is the Kubo number defined as $K=\left(B_{\perp} / B_{0}\right)\left(\lambda_{z} / \lambda_{\perp}\right)$ (Vlad et al. 1998; Balescu 2000b,a; Zimbardo et al. 2000). It characterizes the magnetic turbulence. Its importance stems from the fact that it is the only 
non-dimensional parameter that enters the equation describing the perpendicular transport of magnetic field lines. When the Kubo number is small, i.e. $K \ll 1$, the turbulent transport of field lines is well-described by the quasilinear approximation, which predicts that $D_{m} \approx\left(\lambda_{\perp}^{2} / \lambda_{z}\right) K^{\gamma}$ with $\gamma=2$. This result is identical to the case when the turbulence is slab, i.e. $\lambda_{\perp}=\infty$. In other words, when $K \ll 1$, which is the domain of applicability of the quasilinear approximation, the magnetic diffusion coefficient is independent of $\lambda_{\perp}$ and scales as the second power of the relative level of fluctuations $B_{\perp} / B_{0}$, i.e. $D_{m} \approx \lambda_{z}\left(B_{\perp} / B_{0}\right)^{2}$. From the HXR measurements of $D_{m}$, Kontar et al. (2011) gave constraints on the level of turbulent magnetic fluctuations for a specific event, assuming that the magnetic turbulence is slab or equivalently $K \ll 1$. However, it is established that anisotropy of turbulence, with $\lambda_{\perp} \ll \lambda_{z}$, is prevalent in magnetized plasmas. Moreover, when the Kubo number is large, i.e. $K \gg 1$, the quasilinear theory fails, the magnetic diffusion coefficient no longer scales as $\left(B_{\perp} / B_{0}\right)^{2}$ but instead $D_{m} \approx\left(\lambda_{\perp}^{2} / \lambda_{z}\right) K^{\gamma}$ with $\gamma<1$. Therefore, if we aim to relate measurements of the magnetic diffusion coefficient to the relative level of magnetic fluctuations produced by the turbulence inside flaring loops, we need not only additional observational constraints on the correlation lengths $\lambda_{z}$ and $\lambda_{\perp}$ but also some theoretical predictions on the scaling of $D_{m}$ with $B_{\perp} / B_{0}$ when $K \gg 1$.

Here, we discuss the various regimes of cross-field transport of non-thermal electrons resulting from broadband magnetic turbulence in flaring coronal loops. Results applicable in both the large and small $K$ limits are collected and are applied to the interpretation of hard X-ray imaging data.

\section{Perpendicular motion of energetic electrons in coronal loops}

In the guiding-centre approximation, the perpendicular transport of particles is described by

$$
\frac{\mathrm{d} \boldsymbol{r}_{\perp}}{d t}=v_{z} \frac{\boldsymbol{B}_{\perp}}{B_{0}}+\frac{1}{B_{0}}\left(\boldsymbol{E}_{\perp} \times \boldsymbol{b}_{0}\right)
$$

where the background magnetic field $\boldsymbol{B}_{0}$ is uniform and directed along $\boldsymbol{z}\left(\equiv \boldsymbol{b}_{0}\right), \boldsymbol{B}_{\perp}$ and $\boldsymbol{E}_{\perp}$ are magnetic and electric fluctuations perpendicular to $\boldsymbol{B}_{0}$, and $v_{z}$ is the electron velocity parallel to the guiding field. There are two contributions to the perpendicular transport of particle gyrocentres. One contribution arises from electric field fluctuations, which produce the $\boldsymbol{E} \times \boldsymbol{B}$-drift: $v_{E}=$ $\left(1 / B_{0}\right) \boldsymbol{E}_{\perp} \times \boldsymbol{b}_{0}$. The other contribution comes from the magnetic field fluctuations which also produce a perpendicular drift given by $v_{B}=\mathrm{v}_{z}\left(\boldsymbol{B}_{\perp} / B_{0}\right)$.

The effect of perpendicular electric field fluctuations is negligible for the cross-field transport of non-thermal electrons in coronal loops, which have $v_{\mathrm{Te}} \sim v_{\mathrm{A}}, v_{\mathrm{Te}}$ is the electron thermal speed and $v_{\mathrm{A}}$ the Alfven speed. Indeed for magnetohydrodynamics (MHD) turbulence, $E_{\perp} \sim v_{\mathrm{A}} B_{\perp}$ and therefore $v_{B} / v_{E} \sim v_{z} / v_{\mathrm{A}}$. The neglect of the $\boldsymbol{E} \times \boldsymbol{B}$ drift contribution to perpendicular transport is thus justified provided $v_{z} \gg v_{\mathrm{A}}$. Indeed, Kontar et al. (2011) report $v_{\mathrm{A}} \simeq 1000 \mathrm{~km} \mathrm{~s}^{-1}$ and $v_{z} \simeq 50000 \mathrm{~km} \mathrm{~s}^{-1}$ for electrons producing tens of $\mathrm{keV} \mathrm{X}$-rays.

Because the smallness of the $\boldsymbol{E} \times \boldsymbol{B}$ drift is verified for nonthermal electrons in coronal loops, it means that the cross-field transport is dominated by magnetic fluctuations. As a consequence, the gyrocentre equation of motion simplifies to

$$
\frac{\mathrm{d} \boldsymbol{r}_{\perp}}{\mathrm{d} t}=v_{z} \frac{B_{\perp}}{B_{0}}
$$

In other words, fast electrons in coronal loops tend to follow the field lines because their Larmor radius is small (few centimeters for the coronal parameters) and because their $\boldsymbol{E} \times \boldsymbol{B}$ drift is unimportant. Let us notice, however, that the electric contribution to perpendicular transport becomes of the same order as the magnetic contribution for thermal electrons.

\section{Magnetic field-line transport}

The above discussion shows that the cross-field transport of fast electrons is dominated by the turbulent field-line wandering that is generated through

$\frac{\mathrm{d} \boldsymbol{r}_{\perp}}{\mathrm{d} z}=\frac{\boldsymbol{B}_{\perp}}{B_{0}}$

We emphasize that Eq. (3) has a Hamiltonian structure because $\boldsymbol{B}_{\perp}=\nabla A_{z} \times z$, where $A_{z}$ is the parallel component of the vector potential.

A control parameter of the problem is the Kubo number. This number appears by writing the field-line Eq. (3) in a form obtained after normalizing $r_{\perp}$ and $z$ by $\lambda_{z}$ and $\lambda_{\perp}$, the parallel and perpendicular correlation lengths of the magnetic perturbations. This form is $\mathrm{d} \boldsymbol{r}_{\perp} / \mathrm{d} z=\boldsymbol{K}$ with $\boldsymbol{K}=\left(\lambda_{z} / \lambda_{\perp}\right)\left(\boldsymbol{B}_{\perp} / B_{0}\right)$. Therefore the only non-dimensional parameter entering the equation describing the perpendicular transport of field line is

$K=\frac{B_{\perp}}{B_{0}} \frac{\lambda_{z}}{\lambda_{\perp}}$

which is called the Kubo number (Vlad et al. 1998; Balescu 2000b,a; Zimbardo et al. 2000).

Without loss of generality, we focus on the dispersion of magnetic field lines in the $x$ direction given by

$\frac{\mathrm{d} x}{\mathrm{~d} z}=\frac{B_{x}}{B_{0}}$.

A similar equation for the $y$-displacement involves $B_{y} / B_{0}$ and it is assumed that $B_{y} / B_{0} \sim B_{x} / B_{0}$.

The turbulent field is homogeneous with zero average, $\left\langle B_{x}\right\rangle=0$, where \langle\rangle denotes the ensemble average. The twopoint Eulerian correlation function of the magnetic perturbation is given, it is

$E\left(\boldsymbol{r}_{\perp}, z\right)=\left\langle B_{x}(0,0) B_{x}\left(\boldsymbol{r}_{\perp}, z\right)\right\rangle$.

As an example, we may consider the following form

$E\left(\boldsymbol{r}_{\perp}, z,\right)=B_{x}^{2} \exp \left(-r_{\perp}^{2} / \lambda_{\perp}^{2}\right) \exp \left(-z / \lambda_{z}\right)$

which depends only on two arguments, $r_{\perp}=\left|\boldsymbol{r}_{\perp}\right|$ and $z$, because of the homogeneity and isotropy in the perpendicular plane.

A main goal of the theory is to determine the variation with $z$ of $\left\langle(\Delta x)^{2}\right\rangle, \Delta x$ being the field-line displacement. To this purpose, it is convenient to introduce a running diffusion coefficient, which is defined as $D_{m}(z)=\mathrm{d}\left\langle(\Delta x)^{2}\right\rangle / 2 \mathrm{~d} z$. An important property of this running diffusion coefficient is that it is related to the Lagrangian correlation function by the Taylor formula

$D_{m}(z)=\frac{1}{B_{0}^{2}} \int_{0}^{z} L\left(z^{\prime}\right) \mathrm{d} z^{\prime}$

Here, the notation

$L(z) \equiv\left\langle B_{x}(0) B_{x}(z)\right\rangle$, 
is used for the Lagrangian correlation, where $B_{x}(z) \equiv$ $B_{x}\left(\boldsymbol{r}_{\perp}(z), z\right)$ is obtained through $\boldsymbol{r}_{\perp}(z)$ by integration of the fieldline equations. When the integral in Eq. (8) converges to a nonzero constant in the limit $z \rightarrow \infty$, i.e. $D_{m}(z \rightarrow \infty) \rightarrow D_{m}$, it follows from the definition of the running diffusion coefficient that

$\left\langle(\Delta x)^{2}\right\rangle=2 D_{m} z$

This expression does not determine the magnetic diffusion coefficient $D_{m}$ but simply states that the field-line displacement follows a standard diffusive process. Deviations from the standard diffusive transport can occur whether $D_{m}(z \rightarrow \infty) \rightarrow 0$ or $D_{m}(z \rightarrow \infty) \rightarrow \infty$. For instance, when $\left\langle(\Delta x)^{2}\right\rangle \propto z^{\alpha}$ with $0<\alpha<1$, the field-line transport is said to be sub-diffusive, while for $\left\langle(\Delta x)^{2}\right\rangle \propto z^{\alpha}$ with $\alpha>1$, the transport is superdiffusive.

Although it is the Eulerian correlation $E\left(\boldsymbol{r}_{\perp}, z,\right)$ or the spectrum of magnetic fluctuations that is assumed to be a known function, this is instead the Lagrangian correlation function $L(z)$ that determines the running diffusion coefficient, and hence, also the mean square displacement of the field lines through the Taylor formula (8). Consequently, the whole difficulty of the turbulent transport theory resides in the determination of the Lagrangian correlation function corresponding to a given Eulerian correlation function, for instance of the form (7). A widespread procedure that relates the Lagrangian correlation to the Eulerian one is the Corrsin approximation (see Sect. 3.2). The Corrsin approximation is equivalent to the quasilinear approximation when the Kubo number is small. However, this procedure fails to account accurately for the role of the non-linearity in the field line equation, and in particular trapping effects, which become important when the Kubo number is large.

\subsection{Slab turbulence}

The determination of the magnetic diffusion coefficient is greatly simplified when the magnetic turbulence is slab, i.e. when the magnetic perturbations are a function of $z$ only, because the Eulerian and Lagrangian correlation functions coincide in this case. Therefore the magnetic diffusion coefficient reads

$D_{m}=\frac{1}{B_{0}^{2}} \int_{0}^{\infty} \mathrm{d} z^{\prime}\left\langle B_{x}(0) B_{x}\left(z^{\prime}\right)\right\rangle \simeq \lambda_{z}\left(\frac{B_{x}}{B_{0}}\right)^{2}$,

and this magnetic diffusion coefficient exists provided $\lambda_{z}$ is finite. This is also the well-known expression for the quasilinear diffusion coefficient (Jokipii 1966; Rechester \& Rosenbluth 1978).

\subsection{The Corrsin approximation}

In general, $B_{x}\left(\boldsymbol{r}_{\perp}, z\right)$ is a function of $\boldsymbol{r}_{\perp}$ that makes the field line equation non-linear. As a result it is a difficult task to express the Lagrangian correlation function $L(z)$ (Eq. (9)), which enters the Taylor formula (8) in terms of the known Eulerian correlation function $E(x, y, z)$. By definition, $L(z)=\int \mathrm{d} \boldsymbol{r}_{\perp}\left\langle B_{x}(0,0) B_{x}\left(\boldsymbol{r}_{\perp}, z\right) \delta\left[\boldsymbol{r}_{\perp}-\boldsymbol{r}_{\perp}(z)\right]\right\rangle$ and $E\left(\boldsymbol{r}_{\perp}, z\right)=$ $\left\langle B_{x}(0,0) B_{x}\left(\boldsymbol{r}_{\perp}, z\right)\right\rangle$. The vast majority of turbulent transport theories are based on the assumption that the propagator $\delta\left[\boldsymbol{r}_{\perp}-\boldsymbol{r}_{\perp}(z)\right]$ is equal to its average over the statistics of the fluctuations, i.e. $\delta\left[\boldsymbol{r}_{\perp}-\boldsymbol{r}_{\perp}(z)\right]=\left\langle\delta\left[\boldsymbol{r}_{\perp}-\boldsymbol{r}_{\perp}(z)\right]\right\rangle$. This independence hypothesis goes back to Corrsin (1959) and allows the Lagrangian correlation function to be written as

$L(z)=\int_{-\infty}^{+\infty} \mathrm{d} \boldsymbol{r}_{\perp} E\left(\boldsymbol{r}_{\perp}, z\right) P\left(\boldsymbol{r}_{\perp}, z\right)$

where $P\left(\boldsymbol{r}_{\perp}, z\right) \equiv\left\langle\delta\left[\boldsymbol{r}_{\perp}-\boldsymbol{r}_{\perp}(z)\right]\right\rangle$ is the probability for a fieldline to make a perpendicular excursion from 0 to $\boldsymbol{r}_{\perp}$ over a distance $z$. In the Corrsin approximation the Lagrangian correlation is obtained as a weighted average of the Eulerian correlation that involves the probability distribution function $P\left(\boldsymbol{r}_{\perp}, z\right)$.

When the Kubo number is small, i.e. $K \ll 1$, the right-hand side of the field line Eq. (3) is also small. Hence, it is possible to make the approximation that $P\left(r_{\perp}, z\right) \sim \delta\left(r_{\perp}\right)$. Consequently, it follows from (12) that the Lagrangian correlation is given by the Eulerian correlation around $r_{\perp}=0$ :

$L(z) \sim E(0, z)$.

This is equivalent to the quasilinear approximation (Jokipii 1966; Rechester \& Rosenbluth 1978), which yields the following expression for the magnetic field-line diffusion coefficient:

$D_{m} \simeq \lambda_{z}\left(\frac{B_{x}}{B_{0}}\right)^{2}=\frac{\lambda_{\perp}^{2}}{\lambda_{z}} K^{2}$.

This is just the restatement of Eq. (11). This scaling of the magnetic diffusion coefficient as the second power of the Kubo number is generally valid provided the Kubo number is much smaller than unity, $K \ll 1$, including the case of slab turbulence. The quasilinear diffusion coefficient scales as the second power of the relative level of magnetic fluctuations.

The substitution $\lambda_{z}=\lambda_{\perp}^{2} / D_{m}$ in Eq. (14) provides the following expression for the magnetic diffusion coefficient:

$D_{m} \simeq \lambda_{\perp}\left(\frac{B_{x}}{B_{0}}\right)=\frac{\lambda_{\perp}^{2}}{\lambda_{z}} K$,

a relation which was originally proposed by Kadomtsev \& Pogutse (1979). These scaling of the diffusion coefficient as the first and second power of the Kubo number (first and second power of the relative level of magnetic fluctuations) can both be obtained under the Corrsin independence hypothesis when the probability distribution function $P(x, y, z)$ satisfies the diffusion equation

$\frac{\partial P}{\partial z}=D_{m}\left(\frac{\partial^{2} P}{\partial x^{2}}+\frac{\partial^{2} P}{\partial y^{2}}\right)$,

with the condition that $P\left(r_{\perp}, 0\right)=\delta\left(r_{\perp}\right)$. Indeed, the substitution of the Gaussian solution for $P(x, y, z)$ or $P\left(r_{\perp}, z\right)$, which depends on $D_{m}$, into

$D_{m}=\frac{1}{B_{0}^{2}} \int_{0}^{\infty} \mathrm{d} z \int_{-\infty}^{+\infty} \mathrm{d} x \mathrm{~d} y E(x, y, z) P(x, y, z)$,

provides an integral equation for $D_{m}$. Alternatively, (17) can be written in terms of the spectral energy density of the magnetic fluctuations as

$D_{m}=\frac{1}{B_{0}^{2}} \int \mathrm{d}^{3} \boldsymbol{k}\left|B_{x}\right|_{\boldsymbol{k}}^{2} \frac{D_{m} k_{\perp}^{2}}{\left(D_{m} k_{\perp}^{2}\right)^{2}+k_{z}^{2}}$,

with $\left\langle B_{x}(\boldsymbol{k}) B_{x}\left(\boldsymbol{k}^{\prime}\right)\right\rangle=\left|B_{x}\right|_{k}^{2} \delta\left(\boldsymbol{k}+\boldsymbol{k}^{\prime}\right)$. A characteristic result of this kind of analysis is an implicit relation for $D_{m}$ rather than 
an explicit expression. This procedure was named "renormalization" in the review article by Bykov \& Toptygin (1993), see also Bykov \& Fleishman (2009). The asymptotic limits $K \ll 1$ and $K \gg 1$ of Eq. (17) or (18), recover the scaling of the diffusion coefficient as second and first power of the Kubo number respectively. Indeed, when $D_{m} k_{\perp}^{2} \gg k_{z}$, i.e. $K \gg 1$, the Corrsin approximation gives $D_{m}^{2}=\left(1 / B_{0}^{2}\right) \int \mathrm{d}^{3} \boldsymbol{k}\left|B_{x}\right|_{k}^{2} / k_{\perp}^{2}$. Here, an essential result is that the magnetic diffusion coefficient remains finite and is given by $D_{m} \simeq \lambda_{\perp}\left(B_{x} / B_{0}\right)$, even for a strictly two-dimensional turbulence. An expression that interpolates the $K \ll 1$ and $K \gg 1$ regimes of transport obtained under the Corrsin approximation can be written as

$D_{m}=\frac{\lambda_{\perp}^{2}}{\lambda_{z}} K^{2}(1+K)^{-1}$

which gives $D_{m}=\left(\lambda_{\perp}^{2} / \lambda_{z}\right) K^{2}$ for $K \ll 1$ and $D_{m}=\left(\lambda_{\perp}^{2} / \lambda_{z}\right) K$ for $K \gg 1$.

\subsection{Magnetic field-line trapping}

A major problem with the Corrsin approximation is precisely that it predicts a non-zero diffusion coefficient, independent of $\lambda_{z}$, for 2D turbulence when $K=\infty$. Indeed, a 2D turbulence with $\lambda_{z}=\infty$ is incapable of producing a standard diffusion. The reason is that the field-line equation is fully integrable and that the original Hamiltonian system for 2D perturbations cannot generate stochastic field lines. Particles that follow the field lines and that are released on surfaces $A_{z}(x, y)=$ const. that close on themselves (minima and maxima of the flux function) will remain trapped inside flux tubes. In pure 2D turbulence, the majority of field lines wind around flux tubes, which means that a) particles that follow the field lines stay confined within the flux tubes, b) the mean-square displacement of particles cannot grow with time so that the diffusion coefficient has to be zero.

Nevertheless, even a weak parallel dependence of the turbulence on $z$ is able to produce the opening of the closed contours, which releases field lines and hence particle trajectories in the perpendicular plane; see Fig. 1 in Hauff et al. (2010). A typical trajectory shows an alternation of trapping and perpendicular displacement. These perpendicular displacements occur when particles remain in regions of low absolute values of the flux function, i.e. close to the magnetic separators, and the overall process is a diffusion.

Dimensionally, the scaling of $D_{m}$ with $K$, in the $K \gg 1$ nonlinear limit, has to obey

$D_{m}=\frac{\lambda_{\perp}^{2}}{\lambda_{z}} K^{\gamma}=\left(\delta B_{\perp} / B_{0}\right)^{\gamma} \lambda_{z}^{\gamma-1} \lambda_{\perp}^{2-\gamma}$

with $\gamma<1$, in order for $D_{m}$ to vanish when $\lambda_{z}=\infty$. The first estimate of $\gamma$ for $K \gg 1$ was given by Gruzinov and co-workers based on an analogy with percolation in a stochastic landscape (Isichenko 1992). It yields $\gamma \simeq 2 / 3$; see also the discussion in Milovanov (2009). This value appears to be valid for Eulerian correlation functions that decay sufficiently fast. Balescu and coworkers have developed analytical methods yielding important progress in the statistical theory of transport (Vlad et al. 1998; Balescu 2000b,a). An expression that interpolates the quasilinear and trapping regime of transport can be written as

$D_{m}=\frac{\lambda_{\perp}^{2}}{\lambda_{z}} K^{2}\left(1+K^{4 / 3}\right)^{-1}$,

which gives $D_{m}=\left(\lambda_{\perp}^{2} / \lambda_{z}\right) K^{2}$ for $K \ll 1$ and $D_{m}=\left(\lambda_{\perp}^{2} / \lambda_{z}\right) K^{2 / 3}$ for $K \gg 1$.
The importance of anisotropy and trapping effects in the nonlinear regime of transport was recently discussed in the context of the propagation of solar energetic electrons in the solar wind (Hauff et al. 2010) and for the transport of thermal electrons in solar coronal loops (Bitane et al. 2010). These considerations, which are here applied to the transport of non-thermal electrons in flaring loops, may lead to substantial variations in the value of the turbulence level that is inferred by applying the turbulent transport theory to the interpretation of data.

\section{Application to transport of fast HRX-emitting electrons in thick-target coronal loops}

In a recent work we developed an approach for determining the magnetic diffusion coefficient in thick-target loops. This is based on RHESSI observations and the X-ray visibility analysis (Hurford et al. 2002). Once X-ray visibilities are fitted with Gaussian-curved ellipsoids, the loop sizes clearly reveal (Xu et al. 2008; Kontar et al. 2011) that both the longitudinal (along the guide field) and latitudinal (across-the guide field) extents of the HXR source, $L(\epsilon)$ and $W(\epsilon)$, are increasing functions of the photon energy $\epsilon$. The energy-dependent loop-length is no surprise within a thick-target scenario (Brown 1971) because higher energy electrons can travel farther away from the region where they are accelerated. As a result the HXR source appears to be longer at higher photon energies (Brown et al. 2002), as is often observed in the dense regions of the atmosphere (e.g. Aschwanden et al. 2002; Mrozek 2006; Kontar et al. 2008; Prato et al. 2009; Kontar et al. 2010; Saint-Hilaire et al. 2010).

One important point here is the increase of the HXR source width with energy. This indicates that transport of particles also occurs in the direction perpendicular to the mean magnetic field of the loop. An other important point is that because of their high speed and small Larmor radius, the cross-field transport of electrons is dominated by magnetic fluctuations inside the loop.

While a fast electron emits in the HXR range, it also travels a distance given by $z \simeq \epsilon^{2} / 2 K^{\prime} n$ in the direction along the guide field, $K^{\prime}=2 \pi e^{4} \ln \Lambda, \ln \Lambda \simeq 20$ is the Coulomb logarithm. For an energy independent length $L_{0}$ of the acceleration region, $L(\epsilon)$ is given by Xu et al. (2008) and can be well approximated by

$L(\epsilon)=L_{0}+\alpha_{z} \epsilon^{2}$,

where $\alpha_{z} \simeq 1 /\left(2 K^{\prime} n\right)$. As a result of the perpendicular transport of field lines, the same electrons also make a perpendicular excursion given by $r_{\perp} \simeq \sqrt{2 D_{m} z}$. This produces the increase of $W(\epsilon)$ with energy. $W$ is the sum of the acceleration region width $W_{0}$ and the part due to lateral transport:

$W(\epsilon)=W_{0}+\alpha_{\perp} \epsilon$

where $\alpha_{\perp}=\sqrt{2 D_{m} \alpha_{z}}$. By fitting Eqs. (22), (23) to the observed $L(\epsilon)$ and $W(\epsilon)$, it is possible to determine the values of the parameters that enter these equations and, hence, to obtain the value of the magnetic diffusion coefficient $D_{m}$, i.e. $D_{m}=$ $\alpha_{\perp}^{2} /\left(2 \alpha_{z}\right)$. Kontar et al. (2011) found $D_{m} \simeq 2 \times 10^{7} \mathrm{~cm}$, and also $L_{0} \simeq 2 \times 10^{9} \mathrm{~cm}$ and $W_{0} \simeq 5 \times 10^{8} \mathrm{~cm}$ for the rising phase of the flare.

From this measured value of $D_{m}$ we would ideally like to determine a value of $\delta B / B_{0}\left(\delta B \equiv B_{\perp}\right)$ but this would require independent knowledge of the correlation lengths $\lambda_{z}$ and $\lambda_{\perp}$. Nonetheless we are able to place interesting constraints on all three parameters. First of all, we can take $\lambda_{z}<L_{0}$ and $\lambda_{\perp}<W_{0}$ because $L_{0}$ and $W_{0}$ are of the order of the integral scales of the visible loop. We must have $\delta B / B_{0}<1$ as well, realizing that 


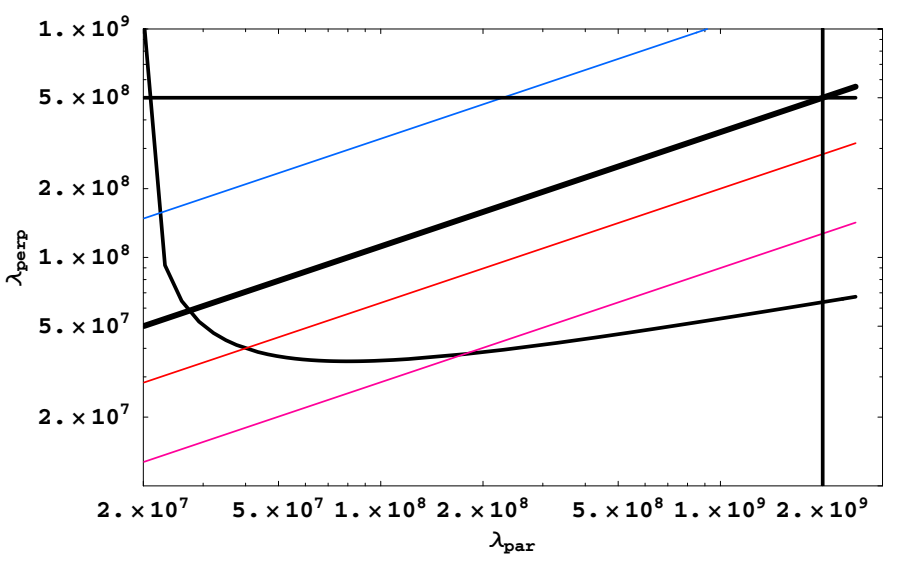

Fig. 1. Kubo number, $K$, in the region of $\left(\lambda_{z}, \lambda_{\perp}\right)$ space allowed by observations, bounded by $\lambda_{\perp}=W_{0}$ (solid horizontal line), $\lambda_{z}=L_{0}$ (solid vertical line) and $\delta B / B_{0}=1$ (solid curve). Kubo number $K$ increases downwards in the figure. The thick black line shows $K=0.467$, and the three coloured lines are drawn for $K=0.14,1$ and 4.67.

magnetic turbulence can power the whole flare for $\delta B \sim B_{0}$; see Kontar et al. (2011). Considering (21) with $\lambda_{z}$ held fixed, we see that a lower limit to $\delta B / B_{0}$ is given by the quasilinear estimate,

$\left(\frac{\delta B}{B_{0}}\right)^{2}=\frac{D_{m}}{\lambda_{z}}$

and thus that $D_{m}<\lambda_{z}<L_{0}$. For a fixed value of $\lambda_{z}$ in this range, $\delta B / B_{0}$ is a decreasing function of $\lambda_{\perp}$ so the requirement $\delta B / B_{0}<1$ now sets a lower limit to $\lambda_{\perp}$, obtainable by rewriting Eq. (21):

$\lambda_{\perp}=\lambda_{z}\left(\frac{\delta B}{B_{0}}\right)\left(\frac{\lambda_{z}}{D_{m}}\left(\frac{\delta B}{B_{0}}\right)^{2}-1\right)^{-4 / 3}$

With $D_{m}$ known and assumed values of $\lambda_{z}$ and $\lambda_{\perp}$, Eq. (21) yields a cubic equation that may be solved exactly for $\left(\delta B / B_{0}\right)^{2}$ (although the resulting expression is not particularly informative). Putting $\lambda_{z}=L_{0}$ and $\lambda_{\perp}=W_{0}$ gives $\delta B / B_{0}=0.12$, the minimum value consistent with $D_{m}$. In Fig. 1 we show the allowed region of $\left(\lambda_{z}, \lambda_{\perp}\right)$ space, bounded by $\lambda_{z}=L_{0}, \lambda_{\perp}=W_{0}$ and $\delta B / B_{0}=1$. From Eq. (24) in the case $\delta B / B_{0}=1$, we can show that $\lambda_{\perp}$ has a minimum value of $4 D_{m} / 3^{3 / 4}=1.75 D_{m}=3.5 \times 10^{7} \mathrm{~cm}$ here.

The energy in perturbations must be at least $\sim 1 \%$ of the energy of the background field. The possibility remains of a much higher energy contained in the turbulent perturbations (see Fig. 2), even sufficient to power the whole flare if $\delta B / B_{0} \simeq 1$. In most of the allowed region $K$ is of the order of unity $0.1 \lesssim K \lesssim$ 10 (see Fig. 1). Nonetheless, we can have significant anisotropy ( $\lambda_{z}$ and $\lambda_{\perp}$ substantially different from one another) without also having $K \gg 1$. Correlation lengths may not, however, be very much less than the natural scales of the magnetic loop.

\section{Discussion and summary}

The theory by Rechester \& Rosenbluth (1978) was applied by Galloway et al. (2006) to the thermal loops observed by the TRACE spacecraft in the extreme ultraviolet range to determine the magnetic turbulence level in thermal loops. The authors found a level of the order of $\delta B / B_{0} \simeq 0.025-0.075$. More recently it was pointed out by Bitane et al. (2010) that by taking into account certain features related to the Kubo number,

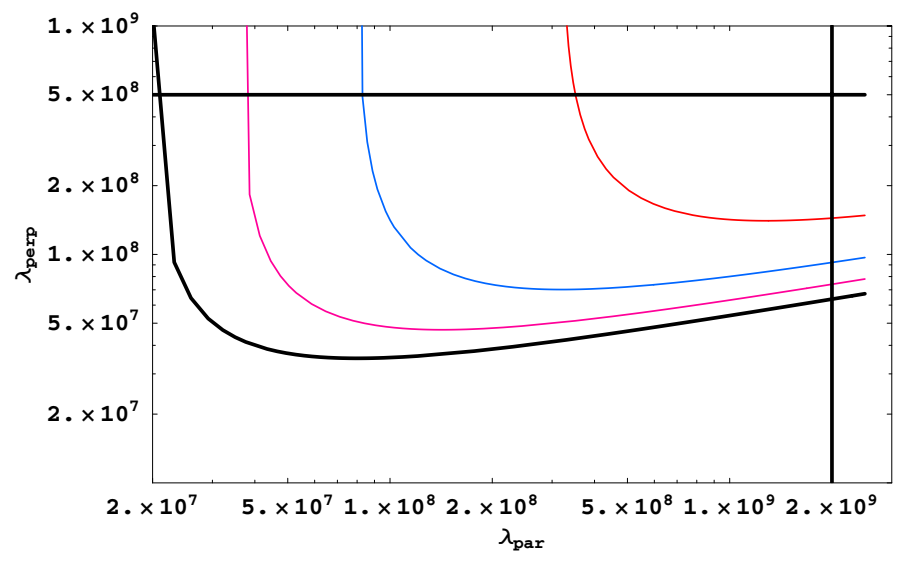

Fig. 2. Contours of $\delta B / B_{0}$ in $\left(\lambda_{z}, \lambda_{\perp}\right)$ space allowed by the observations $\delta B / B_{0}=0.25$ (red), $\delta B / B_{0}=0.5$ (blue) $\delta B / B_{0}=0.75$ (magenta). As in Fig. $1,\left(\lambda_{z}, \lambda_{\perp}\right)$ space bounded by $\lambda_{\perp}=W_{0}$ (solid horizontal line), $\lambda_{z}=L_{0}$ (solid vertical line) and $\delta B / B_{0}=1$ (solid curve) as the limits.

the higher value of the order of $\delta B / B_{0} \simeq 0.05-0.7$ could instead be inferred from the data, values high enough for fieldline braiding to lead to sufficient continuous, small scale reconnection events to account for coronal heating. It should be noted that these estimates for quiet non-flaring loops appear to be as high as the flaring loop estimates. Furthermore, these high values of $\delta B / B_{0} \simeq 0.7$ seem to contradict the observations of non-thermal broadening if interpreted as turbulent velocities. The corresponding MHD turbulence velocities $v \sim B_{\perp} / B_{0} v_{\mathrm{A}} \simeq 50-700 \mathrm{~km} \mathrm{~s}^{-1}$ for $v_{\mathrm{A}} \simeq 1000 \mathrm{~km} \mathrm{~s}^{-1} \mathrm{ap}-$ pear much higher than the typical velocities of tens of $\mathrm{km} \mathrm{s}^{-1}$ inferred from non-thermal line broadening (Doyle et al. 1997; Hara \& Ichimoto 1999; Imada et al. 2009). For the solar flare conditions discussed by Kontar et al. (2011), the non-thermal broadening is instead measured in the range of 100-200 $\mathrm{km} \mathrm{s}^{-1}$ (Antonucci et al. 1982; Doschek 1983; Fludra et al. 1989; Pérez et al. 1999) so, $\delta B / B_{0} \simeq 0.1-0.2$ is indeed consistent with these observations. We note that the thermal particles are more likely to be influenced by $\boldsymbol{E}_{\perp} \times \boldsymbol{B}$ drift, which is dominant for particles whose speeds is comparable to or less than the Alfven speed. The observed appearance of TRACE loops would then constrain $\delta B / B_{0}$ to even lower values than those found by Galloway et al. (2006). Alternatively, the ratio of magnetic and kinetic energies in the turbulence is far from unity and hence the simple relation $v \sim B_{\perp} / B_{0} v_{\mathrm{A}}$ is not applicable.

We considered the cross-field transport of fast electrons inside coronal loops. Our analysis was based on a novel method, which exploits the RHESSI imaging capabilities for determining the value of the magnetic diffusion coefficient in thick target loops. By "thick target" we mean that the flaring loop is dense enough to guarantee that the electrons remain in the loop while they are accelerated and emit HXRs, and hence that they are well-observed with X-ray imaging instruments. Various possible regimes of cross-field transport of non-thermal electrons were discussed and applied to the interpretation of the data. The importance of the Kubo number $K$ as a governing parameter was emphasized and results applicable to both the quasilinear $(K \ll 1)$ and trapping limits $(K \gg 1)$ were collected.

The combination of theory and observation allows us to place interesting constraints on the relative level of magnetic fluctuations and on the Kubo number in flaring loops. These are summarized in Figs. 1, 2. By identifying parallel and perpendicular correlation lengths with the two integral scales of the visible HXR loop, we found $\delta B / B_{0} \simeq 0.1$ and also $K \simeq 0.4$. This 
quasilinear estimate for $\delta B / B_{0}$ shows that magnetic fluctuations with energy of at least $\sim 1 \%$ of the energy of the background field can be inferred from measurements of the magnetic diffusion coefficient.

We note that although the size of the HXR emitting region is likely to be governed by parallel and perpendicular transport, continuing theoretical effort is needed to describe the electron dynamics more accurately, in particular regarding the treatment of the impact of energy loss, acceleration and non-linearity on transport.

Acknowledgements. The authors are grateful to G. Fleishman and V. Nakariakov for valuable discussions. This work is supported by a STFC rolling grant (N.H.B., E.P.K., A.L.M.). Financial support by the European Commission through the HESPE Network is gratefully acknowledged.

\section{References}

Antonucci, E., Gabriel, A. H., Acton, L. W., et al. 1982, Sol. Phys., 78, 107 Aschwanden, M. J. 2002, Space Sci. Rev., 101, 1

Aschwanden, M. J., Brown, J. C., \& Kontar, E. P. 2002, Sol. Phys., 210, 383

Balescu, R. 2000a, Plasma Physics and Controlled Fusion, 42, 1

Balescu, R. 2000b, Eur. J. Phys., 21, 279

Bian, N. H., Kontar, E. P., \& Brown, J. C. 2010, A\&A, 519, A114

Bitane, R., Zimbardo, G., \& Veltri, P. 2010, ApJ, 719, 1912

Brown, J. C. 1971, Sol. Phys., 18, 489

Brown, J. C., \& Kontar, E. P. 2005, Adv. Space Res., 35, 1675

Brown, J. C., Aschwanden, M. J., \& Kontar, E. P. 2002, Sol. Phys., 210, 373

Bykov, A. M., \& Fleishman, G. D. 2009, ApJ, 692, L45

Bykov, A. M., \& Toptygin, I. 1993, Physics Uspekhi, 36, 1020

Chiueh, T., \& Zweibel, E. G. 1987, ApJ, 317, 900

Corrsin, S. 1959, Adv. Geophys., 6, 441

Doschek, G. A. 1983, Sol. Phys., 86, 49

Doyle, J. G., O’Shea, E., Erdelyi, R., et al. 1997, Sol. Phys., 173, 243

Fludra, A., Bentley, R. D., Lemen, J. R., Jakimiec, J., \& Sylwester, J. 1989, ApJ, 344,991
Galloway, R. K., Helander, P., \& MacKinnon, A. L. 2006, ApJ, 646, 615

Hamilton, R. J., \& Petrosian, V. 1992, ApJ, 398, 350

Hara, H., \& Ichimoto, K. 1999, ApJ, 513, 969

Hauff, T., Jenko, F., Shalchi, A., \& Schlickeiser, R. 2010, ApJ, 711, 997

Hurford, G. J., Schmahl, E. J., Schwartz, R. A., et al. 2002, Sol. Phys., 210, 61

Imada, S., Hara, H., \& Watanabe, T. 2009, ApJ, 705, L208

Isichenko, M. B. 1992, Rev. Mod. Phys., 64, 961

Jokipii, J. R. 1966, ApJ, 146, 480

Kadomtsev, B. B., \& Pogutse, O. P. 1979, in Plasma Physics and Controlled Fusion, Research 1978, 1, 649

Kontar, E. P., Hannah, I. G., \& MacKinnon, A. L. 2008, A\&A, 489, L57

Kontar, E. P., Hannah, I. G., Jeffrey, N. L. S., \& Battaglia, M. 2010, ApJ, 717, 250

Kontar, E. P., Hannah, I. G., \& Bian, N. H. 2011, ApJ, 730, L22

Kosugi, T., Masuda, S., Makishima, K., et al. 1991, Sol. Phys., 136, 17

Larosa, T. N., Moore, R. L., \& Shore, S. N. 1994, ApJ, 425, 856

Lin, R. P. 2006, Space Sci. Rev., 124, 233

Lin, R. P., \& Rhessi, T. 2003, Adv. Space Res., 32, 1001

Lin, R. P., Dennis, B. R., Hurford, G. J., et al. 2002, Sol. Phys., 210, 3

Litvinenko, Y. E. 2006, A\&A, 452, 1069

Melrose, D. B. 1994, ApJS, 90, 623

Miller, J. A., \& Ramaty, R. 1987, Sol. Phys., 113, 195

Milovanov, A. V. 2009, Phys. Rev. E, 79, 046403

Mrozek, T. 2006, Adv. Space Res., 38, 962

Pérez, M. E., Doyle, J. G., Erdélyi, R., \& Sarro, L. M. 1999, A\&A, 342, 279

Petrosian, V., \& Chen, Q. 2010, ApJ, 712, L131

Prato, M., Emslie, A. G., Kontar, E. P., Massone, A. M., \& Piana, M. 2009, ApJ, 706, 917

Rechester, A. B., \& Rosenbluth, M. N. 1978, Phys. Rev. Lett., 40, 38

Saint-Hilaire, P., Krucker, S., \& Lin, R. P. 2010, ApJ, 721, 1933

Shibata, K. 1999, Ap\&SS, 264, 129

Somov, B. V., \& Kosugi, T. 1997, ApJ, 485, 859

Vlad, M., Spineanu, F., Misguich, J. H., \& Balescu, R. 1998, Phys. Rev. E, 58, 7359

Xu, Y., Emslie, A. G., \& Hurford, G. J. 2008, ApJ, 673, 576

Zimbardo, G., Pommois, P., \& Veltri, P. 2000, Phys. A Stat. Mech. Appl., 280, 99 